\title{
Treatment with Raltegravir, a retroviral integrase inhibitor, in patients infected with HTLV-1
}

\author{
Ana Treviño $0^{1 *}$, Carmen de Mendoza ${ }^{1}$, Patricia Parra ${ }^{1}$, Jose M Eiros ${ }^{2}$, Raul Ortiz de Lejarazu², Vincent Soriano \\ From 15th International Conference on Human Retroviruses: HTLV and Related Viruses \\ Leuven and Gembloux, Belgium. 5-8 June 2011
}

\section{Background}

The HTLV-1 integrase enzyme shares important structural similarities with the HIV-1 integrase. Experimental studies have demonstrated that styrylquinolines and diketo acids can inhibit both HIV-1 and HTLV-1 integrases. Raltegravir is the only approved integrase inhibitor for the treatment of HIV infection. No data exist about its potential benefit in HTLV-1 carriers.

\section{Methods}

A series of HTLV-1 individuals on regular follow-up at two Spanish clinics were invited to participate in a pilot trial in which raltegravir $40 \mathrm{mg}$ bid was administered for 12 months. Proviral DNA was measured using a validated real-time PCR assay that targets the HTLV-1 pol region on PBMCs at baseline and periodically during follow-up. Demographics and clinical signs/symptoms were closely monitored in all patients.

\section{Results}

A total of 5 HTLV-1 individuals entered the study. Their country of origin was Peru (2), Ecuador (1), Dominican Republic (1) and Colombia (1). Their median age was 52-years old. Three were women. All were infected with HTLV-1 subtype a subgroup a transcontinental. Route of contagion was heterosexual contact in 3 , Transfusion in 1 and homosexual relationships in 1 . The latest was coinfected with HIV-1. Two patients had TSP/HAM whereas the other 3 were asymptomatic.

Median proviral load was 1,658 HTLV-1 DNA copies per 10,000 PBMC in symptomatic patients compared to 758 copies in asymptomatic individuals. Following initiation of raltegravir therapy and up to 6 months, TSP patients experienced a transient decline in HTLV-1 proviral load (from 2,248 to 519 and from 1,033 to 861 copies per 10,000 PBMC, respectively). However, a return to baseline levels was seen in subsequent determinations, being proviral load at 12 months of 2,219 and 1,175 , respectively. No improvement in clinical manifestations could be recognised. No significant changes in HTLV-1 proviral load were noticed in the 3 asymptomatic individuals with median proviral load values over time fluctuating around 755 copies $/ 10,000$ PBMCs.

\section{Conclusions}

Treatment with raltegravir may produce a transient decline in HTLV-1 proviral load in TSP/HAM patients, which is not seen in asymptomatic carriers. However, there is no improvement in TSP/HAM clinical manifestations nor sustained virological benefit beyond six months of therapy.

\section{Author details}

${ }^{1}$ Hospital Carlos III, Madrid, Spain. ${ }^{2}$ Hospital Clínico Universitario, Valladolid, Spain.

Published: 6 June 2011

doi:10.1186/1742-4690-8-S1-A55

Cite this article as: Treviño et al:: Treatment with Raltegravir, a retroviral integrase inhibitor, in patients infected with HTLV-1. Retrovirology 20118 (Suppl 1):A55. 